\title{
Measurement of hadronic form factors at BESIII
}

\author{
Haiming $\mathrm{HU}^{1, \star}$ on behalf of the BESIII Collaboration \\ ${ }^{1}$ Institute of High Energy Physics, CAS, Beijing, China.
}

\begin{abstract}
The measurements of hadronic form factors of three modes using the data samples collected with the BESIII detector at BEPCII collider are presented. The cross section of $e^{+} e^{-} \rightarrow p \bar{p}$ at 12 energies from 2232.4 to $3671.0 \mathrm{MeV}$ are measured, the electromagnetic form factor is deduced, and the ratio $\left|G_{E} / G_{M}\right|$ is extracted by fitting the polar angle distribution. The preliminary results about the form factors of $e^{+} e^{-} \rightarrow \Lambda_{c}^{+} \bar{\Lambda}_{c}^{-}$ will also be described. The cross section of $e^{+} e^{-} \rightarrow \pi^{+} \pi^{-}$between effective center-ofmass energy 600 and $900 \mathrm{MeV}$ is measured by the ISR return method using the data set with the integrated luminosity of $2.93 \mathrm{fb}^{-1}$ taken at $\psi(3773)$ peak, the pion form factor is extracted.
\end{abstract}

\section{Introduction}

The standard model (SM) is the basic theory of elementary particles, its one of the main subjects is to explore the inner structure of hadrons (mesons and baryons). Two or three valence quarks (also sea quarks and gluons) are confined within hadrons, and the interaction among the quarks and gluons are nonperturbative, which leads to the complex multi-parton structures. At present, many properties of hadrons could not be accurately calculated and explained from the first principle of the quantum chromodynamics (QCD). In the study of the strong interaction and hadronic structures, experiments always take crucial role.

BEPCII is a double-ring $e^{+} e^{-}$collider running at center-of-mass energies between 2.0 and $4.6 \mathrm{GeV}$ and reached a peak luminosity of $10^{33} \mathrm{~cm}^{-2} \mathrm{~s}^{-1}$ at center-of-mass energy of $3770 \mathrm{MeV}$. The BESIII detector is located at the BEPCII [1], which is designed to fulfill the requirement of the tau-charm physics [2].

The exclusive hadronic production cross sections in the $e^{+} e^{-}$annihilation are usually expressed as the product of the phase-space factor and form factors. For almost processes, the phase-space factors are ordinary, and the special dynamics reflect in the form factors. From this point of view, if the form factors are derived from theory and confirmed by experiments, it means the laws and properties of strong interactions are understood. One of the quantities can be directly measured in experiments is the (differential) cross section.

Proton and pion are two simplest hadrons composed of the light quarks $\mathrm{u}$ and $\mathrm{d}$, the measurements of form factors to the processes $e^{+} e^{-} \rightarrow p \bar{p}$ and $\pi^{+} \pi^{-}$have special significance. Many experiments did the comprehensive studies to measure the form factors of proton and pion $[3,4]$. BESIII also measured the form factor of strange baryon pair $\Lambda \bar{\Lambda}$, and observed the non-zero cross section near the

^e-mail: huhm@ihep.ac.cn 
threshold [5]. $\Lambda_{c}^{+}$and $\bar{\Lambda}_{c}^{-}$are the grounds of the lightest singly charmed baryons, the properties of the cross section of the process $e^{+} e^{-} \rightarrow \Lambda_{c}^{+} \bar{\Lambda}_{c}^{-}$and $\Lambda_{c}^{ \pm}$decay branch fractions were known less, BESIII did some preliminary measurements using the newly collected data.

This paper will briefly introduce the measurement results of form factors for the following three reaction channels: (1) $e^{+} e^{-} \rightarrow p \bar{p}$ at twelve center-of-mass energies between 2232.4 and $3671.0 \mathrm{MeV}$ [6]; (2) $e^{+} e^{-} \rightarrow \Lambda_{c}^{+} \bar{\Lambda}_{c}^{-}$at energies $4574.5,4580.0,4590.0$ and $4599.5 \mathrm{MeV}$ [7]; (3) $e^{+} e^{-} \rightarrow \pi^{+} \pi^{-}$ between 600 and $900 \mathrm{MeV}$ by the initial state radiation (ISR) return method using the data collected at the peak of $\psi(3773)$ [8].

\section{Measurement of $e^{+} e^{-} \rightarrow p \bar{p}$}

The differential and total Born cross sections of the non-pointlike fermion pair can be expressed as the function of the electromagnetic form factors $\left|G_{E}\right|$ and $\left|G_{M}\right|[9]$,

$$
\begin{aligned}
\frac{d \sigma_{\text {Born }}(s)}{d \Omega} & =\frac{\alpha^{2} \beta C}{4 s}\left[\left|G_{M}(s)\right|^{2}\left(1+\cos ^{2} \theta_{p}\right)+\frac{4 m_{p}^{2}}{s}\left|G_{E}(s)\right|^{2} \sin ^{2} \theta_{p}\right], \\
\sigma_{\text {Born }}(s) & =\frac{4 \pi \alpha^{2} \beta}{3 s} C\left[\left|G_{M}\right|^{2}+\frac{2 m_{p}^{2}}{s}\left|G_{E}\right|^{2}\right] .
\end{aligned}
$$

The meaning of the symbols in above equations are explained in Ref. [6]. The form factors $\left|G_{E}\right|$ and $\left|G_{M}\right|$ can be obtained respectively by fit the measured polar angle $\theta_{p}$ distribution. The earlier experiments measured the $\sigma_{\text {Born }}(s)$ under the simple assumption $\left|G_{E}\right|=\left|G_{M}\right| \equiv|G|$ due to the limited statistics of the data samples. BESIII did the same measurement for a comparison.

Experimentally, the Born cross section of $e^{+} e^{-} \rightarrow p \bar{p}$ is measured by following expression

$$
\sigma_{\text {Born }}=\frac{N_{\mathrm{obs}}-N_{\mathrm{bkg}}}{\mathcal{L} \cdot \varepsilon \cdot(1+\delta)},
$$

where $N_{\mathrm{obs}}$ is the observed number of candidate $p \bar{p}$ pair, $N_{\mathrm{bkg}}$ the number of remnant background events, $\mathcal{L}$ the integrated luminosity, $\varepsilon$ the detection efficiency determined with MC generator, and $(1+\delta)$ the initial state radiation correction factor. The $|G|$ can be deduced with the measured cross section by following relation

$$
|G|^{2}=\frac{3 s}{4 \pi \alpha^{2} \beta C\left(1+2 m_{p}^{2} / s\right)} \sigma_{\text {Born }} .
$$

The measured results by BESIII and other experiments for the invariant $p \bar{p}$ masses from 2.20 to 3.70 $\mathrm{GeV} / c^{2}$ are shown in Figure 1.

Some times, the ratio $R=\left|G_{E} / G_{M}\right|$ is more interested in understanding the electric and magnetic structures, the Eq.(1) can be rewritten as the form:

$$
F\left(\cos \theta_{p}\right)=N_{\text {norm }}\left[1+\cos ^{2} \theta_{p}+\frac{4 m_{p}^{2}}{s} R^{2} \sin ^{2} \theta_{p}\right],
$$

where $N_{\text {norm }}$ is the overall normalization factor. The efficiency corrected distributions of $\cos \theta_{p}$ and fit result for data at c.m. energy $3080.0 \mathrm{MeV}$ of the invariant masse of $p \bar{p}$ is shown in left of Figure 2 . The comparisons of form factor and ratio $\left|G_{E} / G_{M}\right|$ measured with BESIII data to the previous experimental measurements are shown in right of Figure 2. Compared to the BaBar results [10], the precision of the Born cross section measured with BESIII is improved by $30 \%$ for data sets with $\sqrt{s} \leq 3080.0 \mathrm{MeV}$, and the precision of the form factor is improved. 

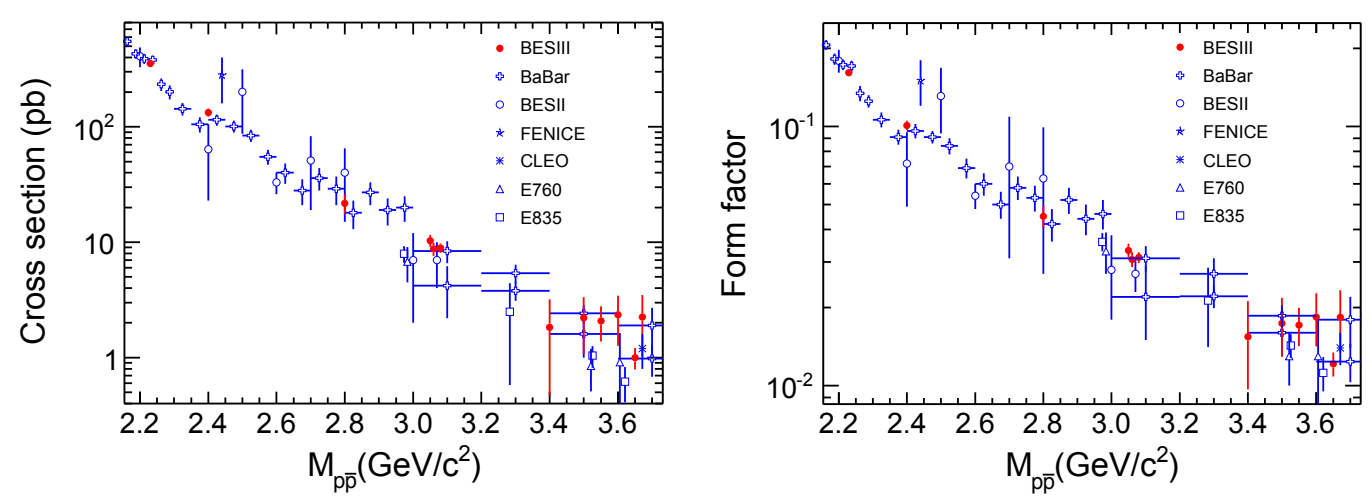

Figure 1. Comparison of the Born cross section (left) and the effective form factor $|G|$ (right) between BESIII measurement and other experiments for the invariant $p \bar{p}$ masses from 2.20 to $3.70 \mathrm{GeV} / c^{2}$.
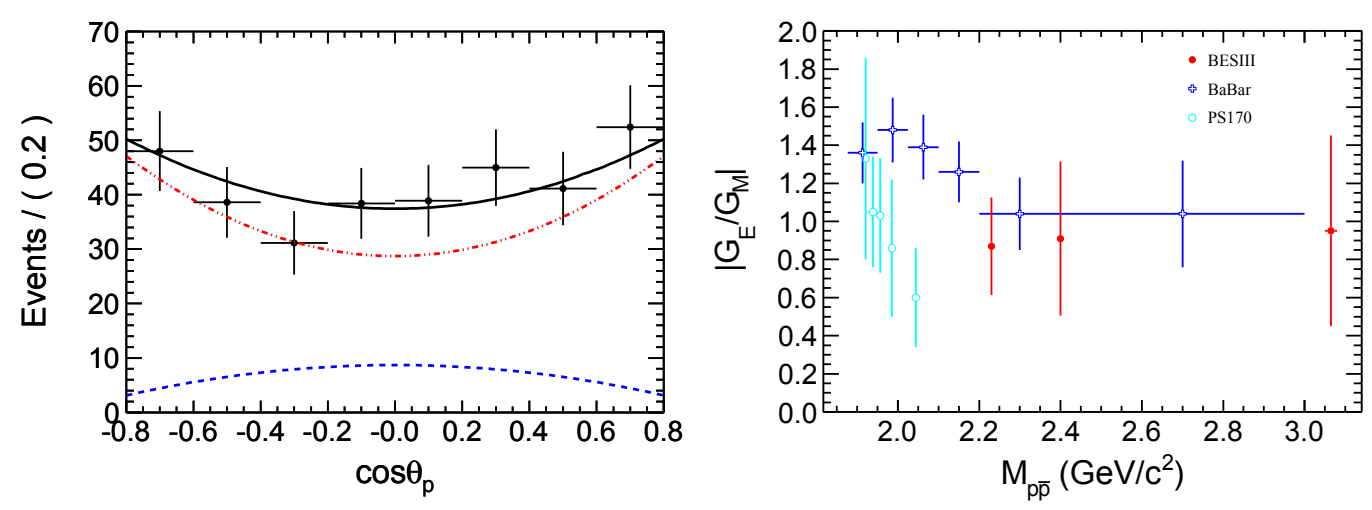

Figure 2. Left: The efficiency corrected distributions of $\cos \theta_{p}$ and fit result for data at c.m. energy $3080.0 \mathrm{MeV}$ of the invariant masse of $p \bar{p}$; Right: the ratio $\left|G_{E} / G_{M}\right|$ at different energies measured by BESIII, BaBar and PS170.

\section{Measurement of $e^{+} e^{-} \rightarrow \Lambda_{c}^{+} \Lambda_{c}^{-}$}

$\Lambda_{c}$ is the lightest single charmed baryon. It was the first hint of charmed baryon in the decay process $\Sigma_{c}^{++} \rightarrow \Lambda_{c}^{+} \pi^{+}$observed at BNL in 1975 [11], and was firstly evidenced at Fermi Laboratory in 1976 [12], then was firstly established at MarkII in 1980 [13]. Most of the heavier charmed baryon will eventually decay to $\Lambda_{c}$, including the doubly charmed baryon $\Sigma_{c c}^{++}$, the error of $B\left(\Lambda_{c}^{+} \rightarrow p K^{-} \pi^{+}\right)$ dominance the uncertainty of $V_{u b}$ via $\Lambda_{b}$ decay.

The form factor of charmed baryon in the process $e^{+} e^{-} \rightarrow \Lambda_{c}^{+} \Lambda_{c}^{-}$near its production threshold is of interested in theory, which leads to various models [14]. Belle Collaboration ever measured the cross section of this channel using initial state radiation (ISR) technique [15]. BESIII also measured the form factor as well as the branching fractions of 12 hadronic decay modes and the semi-leptonic decay $\Lambda_{c}^{+} \rightarrow \Lambda e^{+} v_{e}$ and $\Lambda_{c}^{+} \rightarrow \Lambda \mu^{+} v_{\mu}$ at center-of-mass energy 4574.5, 4580.0, 4590.0 and 4599.5 $\mathrm{MeV}$, the detailed description can be found in references [7]. 


\subsection{The form factor}

The ten Cabibbo-favored hadronic decay modes $\Lambda_{c}^{+} \rightarrow p K^{-} \pi^{+}, p K_{S}^{0}, \Lambda \pi^{+}, p K^{-} \pi^{+} \pi^{0}, p K_{S}^{0} \pi^{0}$, $\Lambda \pi^{+} \pi^{0}, p K_{S}^{0} \pi^{+} \pi^{-}, \Lambda \pi^{+} \pi^{+} \pi^{-}, \Sigma^{0} \pi^{+}$and $\Sigma^{+} \pi^{+} \pi^{-}$, as well as the corresponding charge-conjugate modes are analyzed. The measurement of each mode produce the corresponding cross section, and the total cross section is obtained from the weighted average over the 20 individual measurements [16]. The measured cross section are shown in the left of Figure 3 together with the Belle data [15] for comparisons. The polar angle $\theta$ distribution of the produced fermion pair $\Lambda_{c}^{ \pm}$has the form

$$
f(\theta) \propto\left(1+\alpha_{\Lambda_{c}} \cos ^{2} \theta\right) .
$$

Fitting the angular distribution $f(\theta)$ can be obtained the parameter $\alpha_{\Lambda_{c}}$, and it connects to the ratio $\left|G_{E} / G_{M}\right|$ in following expression:

$$
\left|G_{E} / G_{M}\right|^{2}\left(1-\beta^{2}\right)=\left(1-\alpha_{\Lambda_{c}}\right) /\left(1+\alpha_{\Lambda_{c}}\right) .
$$

The measured angular distribution and the corresponding data fit result at $4599.5 \mathrm{MeV}$ are shown in the right of Figure 3, and the values of fit results of $\alpha_{\Lambda_{c}}$ and $\left|G_{E} / G_{M}\right|$ are listed in Table 1.
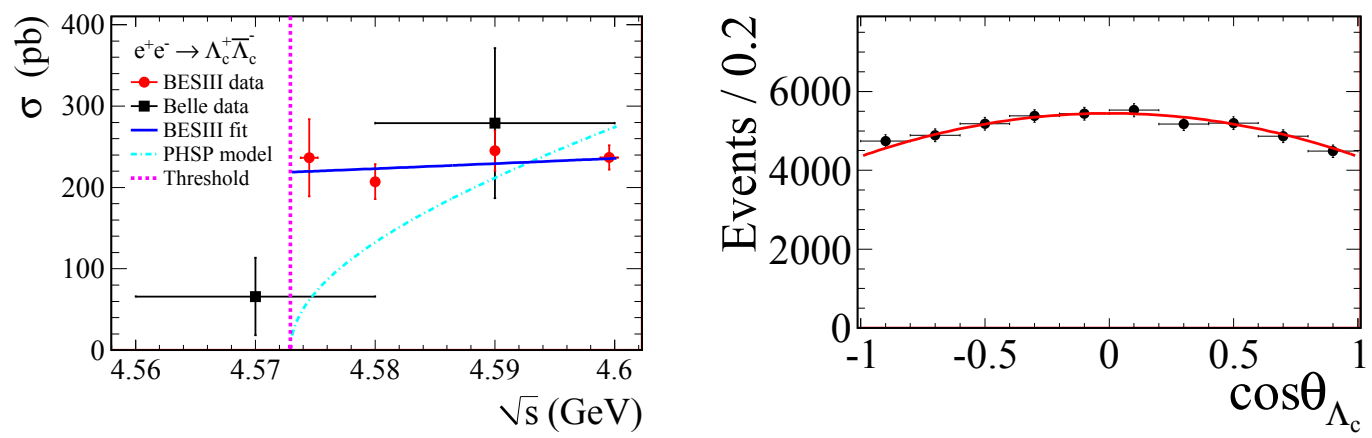

Figure 3. Left: the cross section of $e^{+} e^{-} \rightarrow \Lambda_{c}^{+} \Lambda_{c}^{-}$measured by BESSIII and Belle. The blue solid curve represents the input line-shape for KKMC when determine the $f_{I S R}$. The dash-dot cyan curve denotes the prediction of the phase space model. Right: the angular distribution and the corresponding data fit result at $4599.5 \mathrm{MeV}$.

Table 1. Shape parameters of the angular distribution $\alpha_{\Lambda_{c}}$ and $\left|G_{E} / G_{M}\right|$ for the $e^{+} e^{-} \rightarrow \Lambda_{c}^{+} \Lambda_{c}^{-}$

\begin{tabular}{ccc}
\hline$\sqrt{s}(\mathrm{MeV})$ & $\alpha_{\Lambda_{c}}$ & $\left|G_{E} / G_{M}\right|$ \\
\hline 4574.5 & $-0.13 \pm 0.12 \pm 0.08$ & $1.14 \pm 0.14 \pm 0.07$ \\
4599.5 & $-0.20 \pm 0.04 \pm 0.02$ & $1.23 \pm 0.05 \pm 0.03$ \\
\hline
\end{tabular}

\subsection{The hadronic decay branch ratio}

The 12 Cabibbo-favored hadronic decay modes listed in Table 2 are measured using the data sample collected at $4599.5 \mathrm{MeV}$ with an integrated luminosity of $566.9 \mathrm{pb}^{-1}$. To identify the $\Lambda_{c}^{+} \bar{\Lambda}_{c}^{-}$signal candidates, the tagged techniques $[17,18]$ are adopted, and a least-squares fitter, which considers the statistic and systematic correlations among the different hadronic decay modes, are used to obtain the branch ratio of the 12 decay modes globally. The goodness-of-fit is evaluated as $\chi^{2} / n d f=9.9 /(24-$ 13) $=0.9$, the extracted values are shown in Table 2 . 
Table 2. The preliminary results of the 12 hadronic decay branch ratio of $\Lambda_{c}^{+}$

\begin{tabular}{lcc}
\hline Channel & BESIII preliminary results $(\%)$ & PDG (\%) \\
\hline$p K_{S}^{0}$ & $1.52 \pm 0.08 \pm 0.03$ & $1.15 \pm 0.30$ \\
$p K^{-} \pi^{+}$ & $5.84 \pm 0.27 \pm 0.23$ & $5.0 \pm 1.3$ \\
$p K_{S}^{0} \pi^{0}$ & $1.87 \pm 0.13 \pm 0.05$ & $1.65 \pm 0.50$ \\
$p K_{S}^{0} \pi^{+} \pi^{-}$ & $1.53 \pm 0.11 \pm 0.09$ & $1.30 \pm 0.53$ \\
$p K^{-} \pi^{+} \pi^{0}$ & $4.53 \pm 0.23 \pm 0.30$ & $3.4 \pm 1.0$ \\
$\Lambda \pi^{+}$ & $1.24 \pm 0.07 \pm 0.03$ & $1.07 \pm 0.28$ \\
$\Lambda \pi^{+} \pi^{0}$ & $7.01 \pm 0.37 \pm 0.19$ & $3.6 \pm 1.3$ \\
$\Lambda \pi^{+} \pi^{-} \pi^{+}$ & $3.81 \pm 0.24 \pm 0.18$ & $2.6 \pm 0.7$ \\
$\Sigma^{0} \pi^{+}$ & $1.27 \pm 0.08 \pm 0.03$ & $1.05 \pm 0.28$ \\
$\Sigma^{+} \pi^{0}$ & $1.18 \pm 0.10 \pm 0.03$ & $1.00 \pm 0.34$ \\
$\Sigma^{+} \pi^{+} \pi^{-}$ & $4.25 \pm 0.24 \pm 0.20$ & $3.6 \pm 1.0$ \\
$\Sigma^{+} \omega$ & $1.56 \pm 0.20 \pm 0.07$ & $2.7 \pm 1.0$ \\
\hline
\end{tabular}

\subsection{The semi-leptonic decay branch ratio}

The decay mode to be analyzed is $\Lambda_{c}^{+} \rightarrow \Lambda l^{+} v_{l}$, where $l=e, v$. The candidate of $\bar{\Lambda}_{c}^{-}$is reconstructed with the single tag (ST) by the 11 hadronic decay modes: $\bar{\Lambda}_{c}^{-} \rightarrow \bar{p} K_{S}^{0}, \bar{p} K^{+} \pi^{-}, \bar{p} K_{S}^{0} \pi^{0}, \bar{p} K^{+} \pi^{-} \pi^{0}$, $\bar{p} K_{S}^{0} \pi^{+} \pi^{-}, \bar{\Lambda} K^{-}, \bar{\Lambda} K^{-} \pi^{0}, \bar{\Lambda} K^{-} \pi^{+} \pi^{-}, \bar{\Sigma}^{0} \pi^{-}, \bar{\Sigma}^{-} \pi^{0}$ and $\bar{\Sigma} \pi^{+} \pi^{-}$, where the intermediate particles $K_{S}^{0}, \bar{\Lambda}$, $\bar{\Sigma}^{0}, \bar{\Sigma}^{-}$and $\pi^{0}$ are reconstructed by their following decays: $K_{S}^{0} \rightarrow \pi^{+} \pi^{-}, \bar{\Lambda} \rightarrow \bar{p} \pi^{+}, \bar{\Sigma}^{0} \rightarrow \gamma \bar{\Lambda}$ and $\bar{\Lambda} \rightarrow \bar{p} \pi^{+}, \bar{\Sigma}^{-} \rightarrow \bar{p} \pi^{0}$ and $\pi^{0} \rightarrow \gamma \gamma$. The signal candidates for $\Lambda_{c}^{+}$are selected from the remaining tracks recoiling against the ST $\bar{\Lambda}_{c}^{-}$candidates. The measured results of the branch ratio of the $\Lambda_{c}^{+}$ semi-leptonic decay are

$$
\begin{aligned}
& B_{r}\left(\Lambda_{c}^{+} \rightarrow \Lambda e^{+} v_{e}\right)=(3.63 \pm 0.38 \pm 0.20), \\
& B_{r}\left(\Lambda_{c}^{+} \rightarrow \Lambda \mu^{+} v_{\mu}\right)=(3.49 \pm 0.48 \pm 0.27),
\end{aligned}
$$

which verify the lepton universality in baryon decays within errors:

$$
B_{r}\left(\Lambda_{c}^{+} \rightarrow \Lambda \mu^{+} v_{\mu}\right) / B_{r}\left(\Lambda_{c}^{+} \rightarrow \Lambda e^{+} v_{e}\right)=(0.96 \pm 0.16 \pm 0.04)
$$

\section{Measurement of $e^{+} e^{-} \rightarrow \pi^{+} \pi^{-}$}

\subsection{The theoretical formula}

The cross section of $e^{+} e^{-} \rightarrow \pi^{+} \pi^{-}$in Born (bare) level at the effective center-of-mass energy $\sqrt{s^{\prime}}$ can be expressed in term of the form factor $F_{\pi}\left(s^{\prime}\right)[19,20]$,

$$
\sigma_{\pi \pi}^{0}\left(s^{\prime}\right)=\frac{\pi \alpha^{2} \beta_{\pi}^{3}}{3 s^{\prime}}\left|F_{\pi}\left(s^{\prime}\right)\right|^{2} .
$$

In fact, the measured cross section using the experimental data contains the vacuum polarization and the final state radiation (FSR) effects, the corresponding term in the theory is the so called dressed one $\sigma_{\pi \pi}^{\text {dressed }}$. The bare and dressed cross sections are related by following relation [21]:

$$
\sigma^{\text {bare }}=\frac{\sigma^{\text {dressed }}}{|1-\Pi(s)|^{2}}=\sigma_{\pi \pi}^{\text {dressed }}\left[\frac{\alpha(0)}{\alpha(s)}\right]^{2},
$$

where $\Pi(s)$ is the vacuum polarization factor $[22,23]$. The form factor $\left|F_{\pi}\right|^{2}$ can be extracted using the measured $\sigma_{\pi \pi}^{\text {dressed }}$. 


\subsection{The experimental methods}

The Born cross section of $e^{+} e^{-} \rightarrow \pi^{+} \pi^{-}$was measured by two independent normalization schemes with BESIII data [8].

\subsubsection{The method I}

The following formula is used to measure the Born cross section:

$$
\sigma_{\pi \pi\left(\gamma_{\mathrm{FSR}}\right)}^{\mathrm{bare}}=\frac{N_{\pi \pi \gamma} \cdot\left(1+\delta_{F S R}^{\pi \pi}\right)}{\mathcal{L} \cdot \epsilon_{\mathrm{global}}^{\pi \pi \gamma} \cdot H(s) \cdot \delta_{v a c}},
$$

where $N_{\pi \pi \gamma}$ is the number of signal events, and $H$ the radiator function, $\epsilon_{\text {global }}^{\pi \pi \gamma}$ the global efficiency of the signal, $\delta_{\mathrm{vac}}$ the vacuum polarization correction, $\delta_{\mathrm{FSR}}^{\pi \pi}$ the final state radiation correction factor. The tree-level diagrams for ISR and FSR contribution to $e^{+} e^{-} \rightarrow \pi^{+} \pi^{-} \gamma$ can be found in Ref. [24].

\subsubsection{The method II}

The Born cross section and can also be measured by

$$
\sigma_{\pi \pi\left(\gamma_{\mathrm{FSR}}\right)}^{\mathrm{bare}}=\frac{N_{\pi \pi \gamma}}{N_{\mu \mu \gamma}} \frac{\epsilon_{\mathrm{global}}^{\mu \mu \gamma}}{\epsilon_{\mathrm{global}}^{\pi \pi \gamma}} \frac{1+\delta_{\mathrm{FSR}}^{\mu \mu}}{1+\delta_{\mathrm{FSR}}^{\pi \pi}} \sigma_{\mu \mu}^{\text {bare }} .
$$

In this way, the common factors $\mathcal{L}, H$ and $\delta_{v a c}$ for $e^{+} e^{-} \rightarrow \pi^{+} \pi^{-} \gamma_{I S R}$ and $e^{+} e^{-} \rightarrow \mu^{+} \mu^{-} \gamma_{I S R}$ can be canceled. Where, $\epsilon_{\text {global }}^{\mu \mu \gamma}$ is the global efficiency, $\delta_{\mathrm{FSR}}^{\mu \mu}$ the FSR correction factor, $\sigma_{\mu \mu}^{\text {bare }}$ the bare cross section of $e^{+} e^{-} \rightarrow \mu^{+} \mu^{-}$, which corresponds to a QED process and it can be easily measured in experiment.

\subsection{Data analysis}

The events type $e^{+} e^{-} \rightarrow \pi^{+} \pi^{-} \gamma_{I S R}$ in the invariant mass range $600<m_{\pi \pi}<900 \mathrm{MeV}$ are selected from the data sample taken at center-of-mass energy $\sqrt{s}=3773 \mathrm{MeV}$ [8]. The integrated luminosity of the data set $\mathcal{L}=\left(2931.8 \pm 0.2_{\text {stat }} \pm 13.8_{\text {sys }}\right) \mathrm{pb}^{-1}$. The track-based muon-pion separation based on the Artificial Neural Network (ANN), which is trained using $\pi^{+} \pi^{-} \gamma$ and $\mu^{+} \mu^{-} \gamma$ MC samples, is used to suppress the dominant background $e^{+} e^{-} \rightarrow \mu^{+} \mu^{-} \gamma_{I S R}$. The possible remaining backgrounds are are subtracted using Mont Carlo method.

\subsection{Results}

The results for $\sigma^{\text {bare }}\left(e^{+} e^{-} \rightarrow \pi^{+} \pi^{-}\left(\gamma_{\mathrm{FSR}}\right)\right)$ measured with method I and II are consistent within errors. The cross section and the form factor as the functions of $\sqrt{s^{\prime}}=m_{\pi \pi}$ is illustrated in Fig. 4. The cross section is corrected for vacuum polarization effect, and the final state correction is considered. The result for $\left|F_{\pi}\right|^{2}$ which includes vacuum polarizations, but final state radiation effects are excluded, and the exactly the same fit formula and fit procedure are applied as described in Ref. [4]. Fig. 5 shows the difference between fit and data. The comparisons between the BESIII fit and the previous measurements, BaBar [4], KLOE [25-27]. 

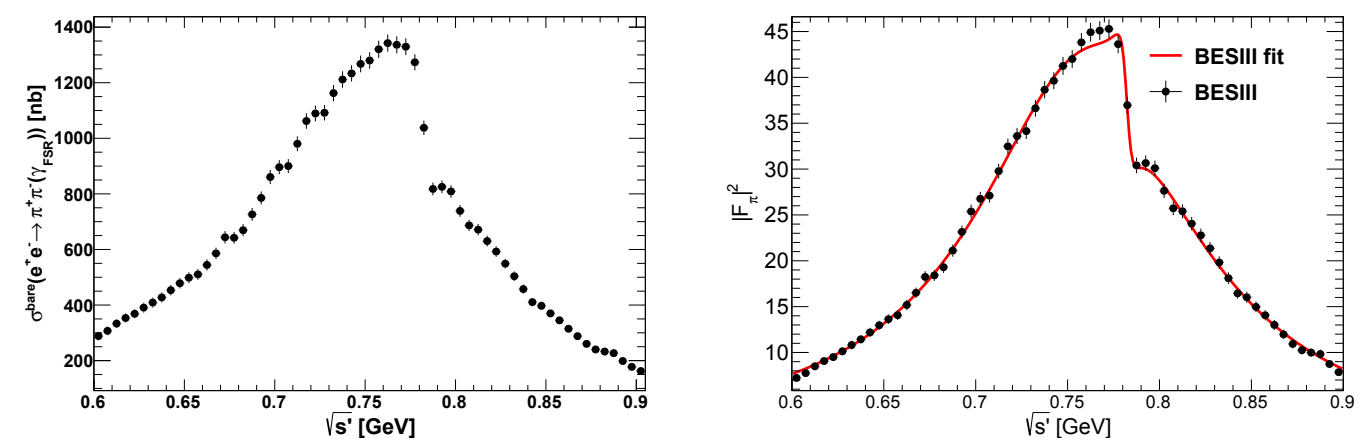

Figure 4. The bare $e^{+} e^{-} \rightarrow \pi^{+} \pi^{-}\left(\gamma_{\mathrm{FSR}}\right)$ cross section (left), and the form factor and the fitted line (right).
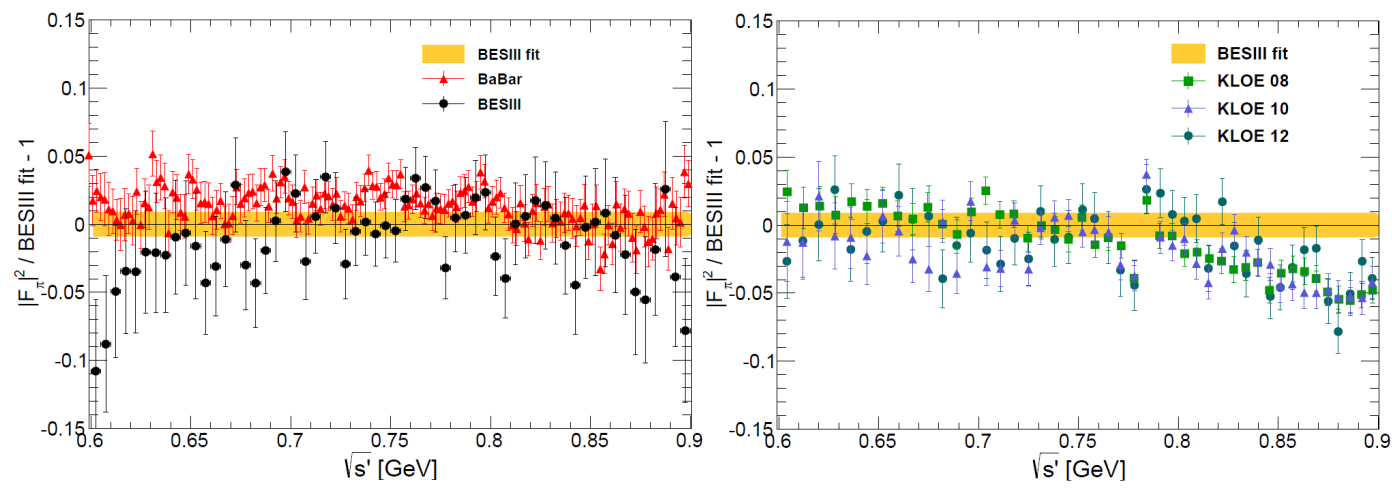

Figure 5. Relative difference of the form factor squared from BaBar (left), KLOE (right) and the BESIII fit. Statistic and systematic uncertainties are included in the data points.

\section{Summary}

The Born cross section of $e^{+} e^{-} \rightarrow p \bar{p}$ is measured, and the effective form factor $|G|$ is extracted under the assumption $\left|G_{E}\right|=\left|G_{M}\right|$ between $2232.4 \mathrm{MeV}$ and $3671.0 \mathrm{MeV}$. The precision of the Born cross section with $\sqrt{s} \leq 3.08 \mathrm{GeV}$ is between $6.0 \%$ and $18.9 \%$. The $\left|G_{E} / G_{M}\right|$ ratios and $\left|G_{M}\right|$ are extracted at the energies $\sqrt{s}=2232.4$ and $2400.0 \mathrm{MeV}$, and a combined data sample with energies of $3050.0,3060.0$ and $3080.0 \mathrm{MeV}$. The measured $\left|G_{E} / G_{M}\right|$ ratios are close to unity which are consistent with the BaBar experiment in the same $q^{2}$ region.

Using the method of ISR return, a measurement of the cross section $\sigma^{\text {bare }}\left(e^{+} e^{-} \rightarrow \pi^{+} \pi^{-}\left(\gamma_{\mathrm{FSR}}\right)\right)$ is performed with an accuracy of $0.9 \%$ in the dominant $\rho(770)$ mass region between 600 and $900 \mathrm{MeV}$. The two-pion contribution to $(g-2)_{\mu}$ is determined to be $a_{\mu}^{\pi \pi, L O}(600-900 \mathrm{MeV})=\left(370.0 \pm 2.5_{\text {stat }} \pm\right.$ $\left.3.3_{\text {sys }}\right) \cdot 10^{-10}$. It is found that a deviation of more than $3 \sigma$ between the SM prediction of $(g-2)_{\mu}$ and its direct measurement is confirmed.

Based on the data sets collected near the production threshold, the Born cross section and electromagnetic form factor ratios of $e^{+} e^{-} \rightarrow \Lambda_{c}^{+} \Lambda_{c}^{-}$are presented. Using the data at $4599.5 \mathrm{MeV}$, the absolute hadronic branching fractions of twelve Cabibbo-favored decays of $\Lambda_{c}^{+}$baryon and the branching fraction of the semi-leptonic decay $\Lambda_{c}^{+} \rightarrow \Lambda l^{+} v_{l}$ were measured. 


\section{References}

[1] M. Ablikim et al. [BESIII Colaboration], Nucl. Instrum. Meth. A 614, 345 (2010).

[2] K. Chao and Y. Wang, Physics at BESIII, Modn. Phys. A Supplement, V24, (2009).

[3] B. Aubert et al. Phys. Rev. Lett. 103, 231801 (2009),

J. P. Lees et al. Phys. Rev. D 86, 032013 (2012).

[4] F. Ambrosino et al. Phys. lett. B 670, 285 (2009),

F. Ambrosino et al. Phys. lett. B 700, 102 (2011),

D. Babusci et al. Phys. lett. B 720, 336 (2013).

[5] M. Ablikim et al. [BESIII Colaboration], arXiv:1709.10236 [hep-ex].

[6] M. Ablikim et al. [BESIII Colaboration], Phys. Rev. D 91, 112004 (2015).

[7] M. Ablikim et al. (BESIII Collaboration) Phys. Rev. Lett. 115, 221805 (2015),

M. Ablikim et al. (BESIII Collaboration) Phys. Rev. B 767, 42 (2017).

[8] M. Ablikim et al. [BESIII Colaboration], Phys. Lett. 753, 629 (2016).

[9] N. Cabibbo and R. Gatto, Phys. Rev. 1241577 (1961).

[10] J. P. Lees et al. (BaBar Collaboration), Phys. Rev. D 87, 092005 (2013)

[11] Phys. Rev. Lett. 34, 1125 (1975)

[12] Phys. Rev. Lett. 37, 882 (1975)

[13] Phys. Rev. Lett. 44, 10 (1980)

[14] O. D. Dalkarov et al. Nucl. Phys. A833, 104 (2010).

B. El-Bennich et al. Phys. Rev. C79, 054001 (2009).

J. Haidenbauer et al. Phys. Lett. B 643, 29 (2006).

R. Baldini et al. Eur. Phys. J. A 39, 315 (2009).

R. Baldini et al. Eur. Phys. J. A 48, 33 (2012).

[15] G. Pakhlova et al. (Belle Collaboration), Phys. Rev. Lett. 101172001 (2008)

[16] M. Schmelling, Phys. Scripta 51, 676 (1995)

[17] R. M. Baltrusaiti et. al. (MARK-III Collaboration), Phys. Rev. Lett. 56, 2140 (1986)

[18] J. Adler et. al. (MARK-III Collaboration), Phys. Rev. Lett. 60, 89 (1988)

[19] B. L. Ioffe, V.A. Khooze, and L.N. Lipatov, Hard processes, Volume 1, Phenomenology QuarkParton Model, (1984), North Holland.

[20] F. E. Close, An Introduction to Quarks and Partons, (1979), Academic Press.

[21] S. Actis et al. arXiv:0912.0749 [hep-ph].

[22] M. E. Peskin and D. V. Schroeder, An Introduction to Quantum field Theory, (1995), Westview Press.

[23] A. Höfer, et al. Eur. Phys. J. C 24, 51, (2002)

[24] S. Pacetti, Nucl. Phys. B (Proc. Suppl.)162, 172(2006).

[25] F. Ambrosino et al. [KLEO Collaboration], Phys. Lett. B 670, 285 (2009).

[26] F. Ambrosino et al. [KLEO Collaboration], Phys. Lett. B 700, 102 (2011).

[27] D. Babusci et al. [KLEO Collaboration], Phys. Lett. B 720, 336 (2013). 